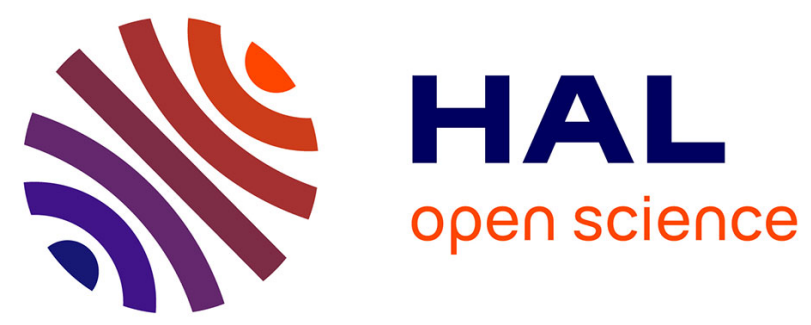

\title{
Les élections européennes en Italie
}

\author{
Carlo Baccetti, Emmanuel Négrier
}

\section{To cite this version:}

Carlo Baccetti, Emmanuel Négrier. Les élections européennes en Italie. Pôle Sud - Revue de science politique de l'Europe méridionale, 1999, Enjeux migratoires enEurope du Sud, 11, pp.116-121. 10.3406/pole.1999.1056 . hal-02549862

\section{HAL Id: hal-02549862 \\ https://hal.science/hal-02549862}

Submitted on 21 Apr 2020

HAL is a multi-disciplinary open access archive for the deposit and dissemination of scientific research documents, whether they are published or not. The documents may come from teaching and research institutions in France or abroad, or from public or private research centers.
L'archive ouverte pluridisciplinaire HAL, est destinée au dépôt et à la diffusion de documents scientifiques de niveau recherche, publiés ou non, émanant des établissements d'enseignement et de recherche français ou étrangers, des laboratoires publics ou privés.

\section{(ㅇ)(1) $\$$}

Distributed under a Creative Commons Attribution - NonCommercial - NoDerivatives $\mid 4.0$ 


\section{Les élections européennes en Italie}

Mr Carlo Baccetti, Mr Emmanuel Négrier

\section{Citer ce document / Cite this document :}

Baccetti Carlo, Négrier Emmanuel. Les élections européennes en Italie. In: Pôle Sud, n¹1, 1999. Enjeux migratoires en Europe du Sud. pp. 116-121;

doi : https://doi.org/10.3406/pole.1999.1056

https://www.persee.fr/doc/pole_1262-1676_1999_num_11_1_1056

Fichier pdf généré le 23/04/2018 


\title{
Les élections européennes en Italie
}

\author{
par Carlo Baccetti \\ Université de Florence \\ (Traduit de l'italien par Emmanuel Négrier) \\ Pôle Sud $N^{\circ} 11$ - novembre 1999 - p. 116 d121
}

Comme il est désormais de tradition, la campagne électorale des élections européennes a été dominée, en Italie, par un débat de politique intérieure. Au cours des cinq dernières années qui ont suivi la précédente consultation, l'enjeu européen a certes acquis une nouvelle dimension dans l'opinion publique italienne, et surtout à la faveur de la monnaie unique. Mais il est toutefois évident que les institutions politiques de cette Europe unie, à commencer par son Parlement, demeurent une thématique assez confuse et peu mobilisatrice pour la majorité du corps électoral.

Ainsi les partis se sont peu appliqués à expliquer aux électeurs le sens de l'activité de leurs représentants à Strasbourg, alors que toute la campagne a été dominée par des choix organisationnels, des mouvements tactiques, des déclarations et interventions polémiques totalement concentrés sur l'arène politique interne.

Les élections européennes étaient inévitablement attendues comme un test du degré de soutien électoral dont jouissait le gouvernement conduit par Massimo D'Alema, au pouvoir depuis l'automne 1998. D'après le leader de l'opposition de centre-droit, Silvio Berlusconi, le gouvernement aurait dû se sentir "moralement" contraint de se démettre si, globalement, les partis de la coalition gouvernementale n'avaient pas atteint un résultat assez positif (que le même Berslusconi avait évalué à $40 \%$ des voix). Au final, le front composite des partis qui sou- tiennent le gouvernement a totalisé $41,2 \%$ (contre $38,1 \%$ au pôle de centre-droit), ce qui a permis à D'Alema de déclarer que le "vote européen ne pose aucun problème de stabilité pour le gouvernement". Il n'y a cependant pas de doute que, au-delà des pourcentages, le test des élections européennes a été tout sauf satisfaisant pour le Président du Conseil, puisqu'il a accentué une fragmentation déjà notable de la majorité parlementaire qui soutient le gouvernement; une fragmentation qui met en péril sinon la stabilité du moins l'efficacité de son action réformatrice.

Les élections européennes sont restées les seules à se tenir selon une proportionnelle intégrale, dont la finalité est de conduire à une proportionnalité maximale dans la répartition des sièges entre listes; il est de fait assez facile aux petites listes d'obtenir un siège. Par conséquent, beaucoup de formations politiques ont interprété ces élections comme la bonne occasion soit pour lancer un nouveau sigle et de nouvelles offres sur le marché électoral, soit pour se démarquer des alliances et faire cavalier seul, en cherchant à étendre ses propres pouvoirs de coalition ou de blocage. Ainsi la campagne électorale a-t-elle été marquée par une forte conflictualité entre partis (une sorte de "tous contre tous") où l'on a eu l'impression que les partis s'employaient davantage à polémiquer au sein de leurs alliances que contre leurs réels adversaires.

Les affrontements se sont en particulier nourris de la présence de deux nouvelles 


\section{Les élections européennes en Italie}

offres électorales dont l'objectif explicite était de remettre en cause les fondements et équilibres propres à la bipolarisation centregauche/centre-droit. A gauche est apparue la nouveauté de I Democratici (les Démocrates), une formation politique crée peu de mois auparavant par Romano Prodi, et à laquelle ont adhéré nombre d'hommes politiques notables, sélectionnés en fonction de leur capacité d'attirer, sur leur seul nom, un grand nombre de voix (de l'ex-magistrat Antonio Di Pietro à quelques maires de grandes villes) : l'objectif déclaré des Démocrates était (et demeure) de peser sur le rapport de force au sein de la coalition. Prodi a en fait considéré qu'il était nécessaire d'améliorer" l'offre électorale de centregauche; son analyse étant que les deux forces principales de la coalition (le PDS de Walter Veltroni et le petit et hyper-gouvernemental Parti Populaire de Franco Marini) se présentent avec une image et une stratégie trop traditionnelles et qu'ils n'ont qu'une faible capacité à faire croître la coalition en termes électoraux. Les Démocrates de Prodi se proposent donc comme la nouvelle pierre angulaire d'une coalition rénovée, opérant à l'intérieur d'un système réellement bipolaire et même bipartisan à plus long terme (avec une sympathie pour une future forme de gouvernement de type présidentiel, comme le laisse clairement entendre le recours à un signe et à un symbole - l'Âne - emprunté au système politique américain).

Au centre-droit, Gianfranco Fini, leader de l'Alleanza Nazionale, et Mario Segni (exparlementaire Démocrate-Chrétien qui avait acquis dans l'opinion publique une image d'innovateur politique) ont tenté une opération symétrique à celle lancée par Prodi à gauche. Les deux hommes ont en fait unifié leurs symbôles respectifs
- Alleanza Nazionale et Patto Segni - sous l'enseigne d'un nouveau : l'Éléphant, lui aussi emprunté à la scène politique américaine. Comme l'Âne de centre-gauche, l'Éléphant était une proposition électorale qui voulait en substance modifier les équilibres et la stratégie politique du Pôle de centre-droit auquel il se rattachait. Fini et Segni défendent en fait l'utilité, pour l'Italie, d'atteindre un modèle bipolaire et présidentialiste et, à ce propos, sont entrés directement en concurrence électorale avec le principal partenaire de l'Alleanza Nazionale, Sivlio Berlusconi, leader de Forza Italia et principal "activiste" du pôle.

L'autre nouveauté importante de cette compétition électorale est la présence de la Liste Emma Bonino. Avec elle, l'ex-commissaire européenne se plaçait explicitement en marge critique des deux principaux courants (même si, de fait, ses attaques se sont concentrées presque exclusivement sur la coalition de centre-gauche au pouvoir). Loffre électorale de la Bonino se présentait comme l'énième "voix des citoyens" contre la partitocratie et se proposait de recueillir un vote protestataire en provenance des tous les secteurs de l'électorat, au-delà des clivages droite-gauche.

\section{Les résultats}

Grande fragmentation électorale et faible participation : ce sont de manière très synthétique les tendances les plus significatives que l'on peut repérer dans ce scrutin européen. Le mode proportionnel, comme nous l'avons déjà dit, a favorisé l'émergence d'une offre très vaste : 26 listes se sont présentées (pour un total de 30 formations politiques), dont 20 étaient présentes sur les cinq circonscriptions qui subdivisaient le territoire 


\section{Pôle Sud $N^{\circ} 11$}

national. A la grande fragmentation de l'offre a correspondu une même fragmentation des suffrages exprimés. Seules trois listes ont dépassé les $10 \%$ des voix (une de peu), et deux ont franchi la barre des $5 \%$; cependant que toutes les autres sont restées inférieures à ce seuil - et la grande partie de ceux-ci se sont limitées à moins de $2 \%$. Dixhuit partis ont obtenu au moins un siège.

La basse participation électorale est le deuxième constat : Seuls $69,8 \%$ des inscrits se sont déplacés aux urnes, soit $4 \%$ de moins que lors des élections de 1994. En pratique, cela représente 1300000 électeurs de moins vis-à-vis de 1994, alors que le corps électoral s'est dans le même temps accru de 800000 inscrits. Plus, on remarque, parmi ceux qui se sont déplacés aux urnes, une croissance du "non-vote" (nul ou blanc) : il représente $9,5 \%$ (avec environ $50 \%$ de votes blancs) en 1999 , contre $7,6 \%$ en 1994 . Ce sont donc seulement $63,1 \%$ des électeurs qui ont exprimé un vote en faveur de l'une des listes en compétition. C'est indiscutablement un taux faible, rapporté à la tradition italienne; même si la chute de la participation est un phénomène continu depuis le début des années 1990, valable pour toutes les consultations et dans toutes les régions, avec il est vrai certains contrastes.

Une rapide analyse du vote démontre (tableau $n^{\circ} 1$ ) des changements importants par rapport aux précédentes élections européennes de 1994 et législatives de 1996 (on doit bien sâr prendre ces comparaisons entre divers types de consultation avec prudence); de même on peut observer des différences significatives des votes en faveur des principaux partis - ceux qui ont franchi la barre des $4 \%$ - d'une zone à l'autre du pays (tableau $n^{\circ} 2$ ).
Les Démocrates de Gauche perdent beaucoup de voix; en s'établissant à 17,3\%, ils perdent le statut de premier parti italien. Il ne fait aucun doute que le parti du Président du Conseil ait subi le contrecoup de l'entrée en lice des Démocrates de Prodi, qui lui ont pris des voix partout de façon homogène, sauf peut-être dans la III' circonscription. En fait, dans les (ex) régions rouges de l'Italie centrale, le PDS tiennent mieux leurs positions que dans les autres zones (au Nord comme au Sud), où la chute du parti est proportionnellement plus important. En Toscane, par exemple, il obtient un acceptable 31,9\%; l'électorat PDS s'est montré moins infidèle et les Démocrates (de Prodi) sont restés en dessous de leur moyenne nationale avec 5,1\%.

En dehors du PDS, l'autre protagoniste principal de la compétition électorale, le Parti Populaire, a été durement affecté. La présence au gouvernement ne suffit pas à protéger le parti d'un recul d'influence sauf dans les circonscriptions méridionales et insulaires, où le PPI peut compter sur des ressources politiques de type clientélaire qui lui ont permis un relatif maintien - qui menace sa propre survie comme force organisée et politiquement importante dans presque tout le Nord du pays. Un sondage réalisé par l'institut IPSO pour le Corriere Della Sera à l'issue des élections a montré qu'environ $20 \%$ des électeurs qui ont choisi l'Ane proviennent autant du PDS que du PPI (alors que les votes soustraits au Pôle de centre-droit ne représentent que moins de $15 \%$, et que les anciens abstentionnistes comptent pour $17 \%$ ).

Le succès de l'Âne de Prodi n'a pas été en revanche suivi par un même essor de l'Éléphant de Fini et Segni qui, au centre-droit, ne sont pas parvenus à écorner la suprématie 


\section{Les élections européennes en Italie}

1. Italie. Elactions europénmes du 13 juin 1999.

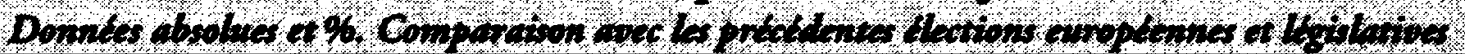




\section{Pôle Sud $N^{\circ} 11$}

\begin{tabular}{c|ccccccccc}
\hline \multicolumn{7}{c}{$\begin{array}{c}\text { 2. Italie. Elections européennes du 13 juin 1999. } \\
\text { Scores des principaux partis dans les différentes circonscriptions électorales }\end{array}$} \\
\hline $\begin{array}{c}\text { Circons- } \\
\text { cription }\end{array}$ & $\begin{array}{c}\text { Forza } \\
\text { Italia }\end{array}$ & DS & $\begin{array}{c}\text { AN } \\
\text { Patto } \\
\text { Segni }\end{array}$ & $\begin{array}{c}\text { Lista } \\
\text { Bonino }\end{array}$ & $\begin{array}{c}\text { I } \\
\text { Demo- } \\
\text { cratici }\end{array}$ & $\begin{array}{c}\text { Lega } \\
\text { Nord }\end{array}$ & PRC & PPI \\
I & 29,5 & 14,0 & 6,7 & 12,0 & 7,2 & 10,5 & 4,3 & 2,5 \\
II & 23,1 & 19,8 & 8,3 & 10,3 & 8,1 & 6,9 & 3,7 & 3,0 \\
III & 20,2 & 24,5 & 15,5 & 7,5 & 6,2 & 0,4 & 5,9 & 3,8 \\
IV & 25.0 & 14,8 & 11,2 & 4,7 & 9,2 & 0,2 & 3,9 & 6,8 \\
V & 27,5 & 12,9 & 12,1 & 5,0 & 8,2 & 0,1 & 2,9 & 7,0 \\
ITALIE & 25,2 & 17,3 & 10,3 & 8,5 & 7,7 & 4,5 & 4,3 & 4,2 \\
\hline
\end{tabular}

I circoscrizione - Italia nord-occidentale : Piemonte, Valle d'Aosta, Liguria, Lombardia

II circoscrizione - Italia nord-orientale : Veneto, Trentino Alto-Adige, Friuli Venezia-Giulia, Emilia-Romagna

III circoscrizione - Italia centrale : Toscana, Umbria, Marche, Lazio

IV circoscrizione - Italia meridionale : Abruzzi, Molise, Campania, Puglia, Basilicata, Calabria

$\mathrm{V}$ circoscrizione - Italia insulare : Sicilia, Sardegna

et le leadership de Berlusconi. L'Alleanza Nazionale a subi une perte de quasiment $5 \%$ par rapport aux dernières élections et s'est à nouveau affirmé comme un parti enraciné avant tout au Centre-Sud, où il a toutefois perdu de l'influence, sans réussir à conquérir de nouveaux espaces électoraux, en particulier dans les zones productives du Nord, politiquement décisives.

Après les élections européennes, le premier parti italien est redevenu Forza Italia $(25,2 \%)$ : le parti de Berlusconi enregistre une nette progression et obtient ses meilleurs résultats dans deux zones politiquement, socialement et économiquement aussi diverses que le Nord-Ouest moderne et "européen" et la circonscription méditerranéenne, sicilienne et sarde.

La Lega Nord de Umberto Bossi, Rifondazione Comunista et Lista Bonino sont les trois listes majeures qui ne font pas partie des coalitions dominantes. Parmi celles-ci, seule la nouvelle, celle de Bonino (en réalité l'ultime et brillante transfiguration de la liste radicale de Marco Pannella), a obtenu un succès notable, particulièrement accentué dans les zones politiquement plus anxieuses du Nord. Les sondages confirment que Bonino et Pannella sont parvenus, comme ils l'escomptaient, à intercepter des électeurs provenant d'un peu toutes les formations politiques, outre l'abstentionnisme.

En revanche, les deux autres forces "antisystème" ont chuté de moitié ou pire par rapport aux précédentes élections législatives ou européennes. L'insuccès électoral de la Lega Nord est dû avant tout au fait que le mot d'ordre du séparatisme (pour "libérer le Nord vertueux et productif d'un Sud abusif et parasitaire et maintenu par l'Etat grâce aux impôts payés par le Nord") n'a pas eu de prise sur l'électorat septentrional, et même pas sur la partie de celui-ci qui avait auparavant montré sa sympathie pour Bossi.

Enfin, en ce qui concerne l'insuccès de Rifondazione Comunista, il est certainement 


\section{Les élections européennes en Italie}

attribuable au choix suicidaire assumé par son secrétaire, Fausto Bertinotti, quand il a décidé en octobre dernier de rompre avec sa stratégie de soutien externe et donc de faire tomber le gouvernement de centre-gauche alors conduit par Romano Prodi. Beaucoup d'électeurs de gauche n'ont pas partagé ce choix de le faire chuter et de donner ainsi un espace plus favorable à l'alliance de centredroit autour de Berlusconi.

En conclusion, le résultat de ce scrutin ne se prête guère à une lecture unique. Probablement l'offre électorale si abondante a-telle troublé ou irrité encore davantage bien des électeurs qui, en nombre croissant, ont décidé de s'abstenir. Les votants ont dispersé leurs choix dans un spectre assez large de partis, même s'ils ont semblé globalement attirés par la "nouveaute" en tant que telle. Ce résultat a été souvent interprété comme une indication de l'opinion politique en faveur de la simplification bipolaire du système politique. C'est en ce sens que l'on devrait lire, selon bien des commentateurs, les constats suivants :

- le bon résultat des Démocrates de Prodi qui soutiennent comme on l'a vu une réforme institutionnelle d'orientation majoritaire et bipolaire;

- le succès de la Liste Bonino qui voudrait en fait introduire en Italie le modèle, présidentiel et bipartisan, des États-Unis;

- l'échec de deux partis qui soutiennent au contraire le maintien du système proportionnel (Rifondazione Comunista et Lega
Nord), qui semblerait indiquer un consensus diffus pour une simplification majoritaire.

On notera cependant que la liste de l'Éléphant, qui s'était exprimé en faveur de la même proposition majoritaire et bipolaire, n'a pas eu un même succès.

Il est vrai qu'en Italie, au cours des années 1990 , un vaste sentiment anti-partisan et un "rejet de la politique" ont pris naissance. Ceux-ci, quand ils ne se traduisent pas par une abstention accrue, tendent à s'illustrer par un soutien confus et indistinct à "nouveau" contre l'ancien"; une alternative que beaucoup aimeraient voir remplacer l'actuel clivage droite-gauche. Dans ce contexte, les élections européennes ont souffert d'une contradiction évidente qui a émergé des urnes : si les partis "nouveaux" les plus fervents de la logique majoritaire ont pu se présenter et se positionner avec succès, ce n'est que grâce au mécanisme hyper-proportionnel qui encadre ces élections européennes. L'exemple le plus évident est celui de la Liste Bonino. Avec elle, en effet, l'électorat a choisi une liste nouvelle et "majoritariste" qui venait pourtant accroître la fragmentation proportionnaliste de l'offre et, par dessus tout, refusait programmatiquement d'être rattaché soit à l'une soit à l'autre des coalitions dominantes; c'est-à-dire une position qui, dans les faits, est la plus patente négation de la logique bipolaire.

A la lumière d'une telle contradiction intrinsèque, il ne nous semble pas que ces élections européennes aient fortement contribué à rendre plus clair et plus proche d'une issue stable, la longue transition politique italienne. 